
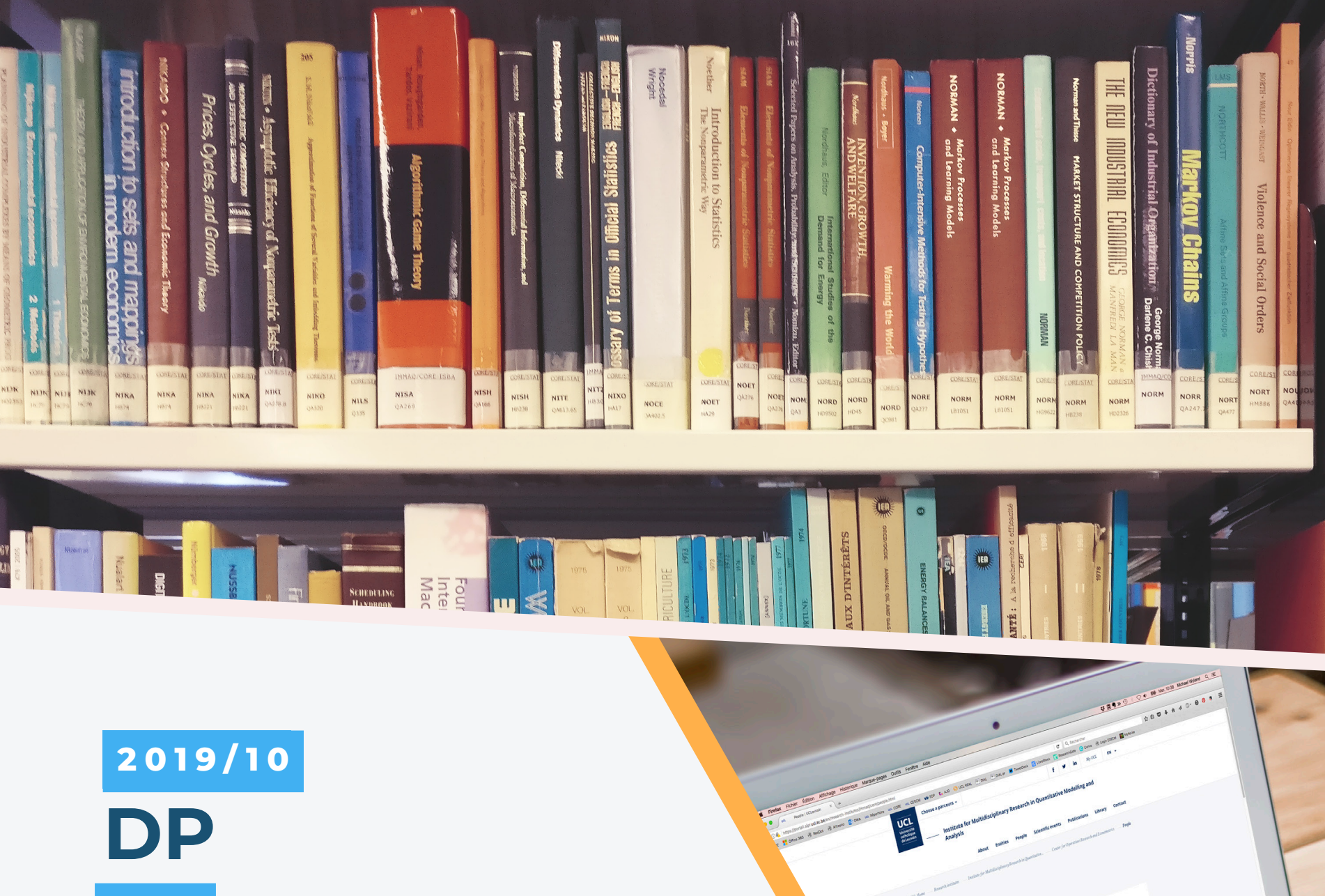

Irinel Dragan and Pierre Dehez

Alternative representation

of semivalues, the inverse

problem and coalitional

rationality 


\section{CORE}

Voie du Roman Pays 34, L1.03.01

B-1348 Louvain-la-Neuve

Tel (32 10) 474304

Email: immaq-library@uclouvain.be

https://uclouvain.be/en/research-institutes/

lidam/core/discussion-papers.html 


\title{
Alternative Representation of Semivalues, the Inverse Problem and Coalitional Rationality
}

\author{
Irinel Dragan * and Pierre Dehez ${ }^{* *}$
}

\begin{abstract}
The concept of semivalue of a transferable utility game has been introduced by Dubey, Neyman and Weber as weighted sum of marginal contributions. Later, Puente has introduced a particular class of semivalues, called binomial semivalues, where weights are obtained through a recursive procedure. In the present paper, we extend Puente's procedure to obtain an equivalent representation of semivalues that turns out to be useful to solve the inverse problem and the question of coalitional rationality.
\end{abstract}

Keywords Transferable utility games, Semivalues, Inverse problem, Power game.

Mathematics Subject Classification 91A12

JEL Classification: C71

* University of Texas, Department of Mathematics

411 S.Nedderman Drive, Arlington, Texas 78019-0408, USA.

E-mail: dragan@uta.edu

** University of Louvain, Center for Operations Research and Econometrics

34 Voie du Roman Pays, 1348 Louvain-la-Neuve, Belgium.

Email: pierre.dehez@uclouvain.be

The authors are grateful to Yurii Nesterov for his comments on an earlier draft. 


\section{Introduction}

A transferable utility game specifies who are the players and what they can achieve when they form coalitions. If there is a potential gain for the players to get together and cooperate, the question that comes up immediately is how to divide this gain, that is how to remunerate the players for their participation to the collective endeavor.

In 1953, Lloyd Shapley introduced axiomatically the notion of value of a transferable utility game that specifies a rule for distributing the gain that the "grand coalition" can generate. A natural question is then the following: Given an allocation, can we identify the set of games whose Shapley value coincides with that allocation? This is the inverse problem that has first been addressed by Kleinberg and Weiss (1985) and Dragan (1991).

"At the foundation of the theory of games is the assumption that a player can evaluate in terms of his utility scale every situation that can result from a play of a game." This quotation from Shapley (1951) indicates that the concept of value was initially intended to answer the question of what a player may reasonably expect from playing a game. That point of view raises the question of efficiency: Are players' evaluations consistent? By requiring that the total gain be exactly allocated among the players, the Shapley value is actually efficient. Dropping efficiency opens the way to other classes of value, among which the semivalues introduced by Dubey, Neyman and Weber (1981). A semivalue allocates to players a weighted sum of their (marginal) contributions to coalitions. The weights only depend on coalition size and define a particular semivalue. The Shapley value happens to be the only efficient semivalue. The Banzhaf value is another well-known semivalue derived from the power index introduced by Banzhaf (1965). The inverse problem for the Banzhaf value has been addressed by Dragan (1996) and, later, for semivalues, by Dragan (2005).

The core introduced by Gillies (1953) is an other game theoretic concept that applies to transferable utility games. The core of a game is the set of efficient allocations such that no coalition globally receives an amount short of what it is worth. This is the question of coalitional rationality. Games often cover situations where it is impossible to satisfy all coalitions i.e. the core of a game may be empty. Furthermore, it carries no value judgment and therefore, when nonempty, it may contain unfair allocations: Coalitional rationality and fairness may be mutually inconsistent. For a given set of weights, looking at the set of games whose semivalue coincides with a particular allocation, we may ask whether there are games for which that allocation belongs to their cores. As such, that question makes sense only if efficiency applies. It has been addressed for the Shapley value by Izawa and Takahashi (1998). If instead efficiency does not apply, coalitional rationality is analyzed with respect to the core of an auxiliary game, the power game, a concept introduced by Dragan (1996) in relation to the Banzhaf value. Dragan and Martinez-Legaz (2001) have later generalized the analysis to semivalues. In the present paper, we reconsider the solution to the inverse problem for semivalues and the 
associated question of coalitional rationality, using an equivalent representation of semivalues inspired by the concept of binomial semivalue introduced by Puente (2000). ${ }^{1}$

The paper is organized as follows. Section 2 introduces semivalues and their equivalent representation. Section 3 revisits the solution of the inverse problem using the notion of potential. Section 4 addresses the question of coalitional rationality. The last section offers concluding remarks.

\section{Semivalues}

A transferable utility game (hereafter "game") is a pair $(N, v)$ where $N=\{1, \ldots, n\}$ is a set of players and $v$ is a set function (a "characteristic function") that associates a real number $v(S)$ to each subset (coalition) $S$ of $N . v(S)$ is the worth of coalition $S$, the best it can obtain by itself. By convention $v(\varnothing)=0$. We denote by $G$ the set of all games. A characteristic function on a set $N$ is defined by $2^{n}-1$ numbers. Hence, the set $G(N)$ of games on a player set $N$ can be identified to the Euclidian space $\mathbb{R}^{2^{n}-1}$. A game $(N, v)$ is additive if $v(S)=\sum_{i \in S} v(i)$ for all $S \subset N$. A game is monotonic if $S \subset T \Rightarrow v(S) \leq v(T){ }^{2}$

Notation: Set inclusion is denoted by $\subset$ and strict inclusion by $\varsubsetneqq$. Upper-case letters denote finite sets and the corresponding lower-case letters denote their sizes: $s=|S|, t=|T|, \ldots$ For a given coalition $S, S \backslash i$ denotes the coalition obtained by removing player $i$ from coalition $S$. For a coalition $T, \mathcal{C}_{i}(T)=\{S \subset T \mid i \in S\}$ denotes the collection of subsets of $T$ containing player $i$. We occasionally drop the braces to denote coalitions and omit the index set in summations when no confusion may arise.

The allocation of $v(N)$ - the worth of the "grand coalition" - among the players is the central question addressed by the theory of cooperative games. A payoff vector $x \in \mathbb{R}^{n}$ specifies a payoff for each player, $x_{i}$ for player $i$. We will consider the following three properties of payoff vectors:

Efficiency: $\sum_{i \in N} x_{i}=v(N)$.

Individual rationality: $x_{i} \geq v(i)$ for all $i \in N$.

Coalitional rationality: $\sum_{i \in S} x_{i} \geq v(S)$ for all $S \subset N$.

Efficiency is a matter of collective rationality. Imputations are payoff vectors satisfying efficiency and individual rationality. The core is the set of imputations that satisfy coalitional rationality. Given a player set $N$, a value is a mapping $\varphi: G(N) \rightarrow \mathbb{R}^{n}$ that associates a payoff vector $\left(\varphi_{1}(N, v), \ldots, \varphi_{n}(N, v)\right)$ to every game $(N, v)$. Semivalues form a family of values

\footnotetext{
${ }^{1}$ The inverse problem for binomial semivalues has been addressed by Dragan (2014).

${ }^{2}$ For more details on transferable utility games, see for instance Owen (1995) or Maschler et al. (2013). Formally a game is a pair $(N, v)$. When $N$ is known, a game is identified with its characteristic function.
} 
introduced by Dubey, Neyman and Weber (1981) as mappings satisfying the following four properties:

Linearity: $\varphi(N, \alpha v+\beta w)=\alpha \varphi(N, v)+\beta \varphi(N, w)$ for all $v, w \in G(N)$ and $\alpha, \beta \in \mathbb{R}$.

Anonymity: $\varphi(N, \pi v)=\pi \varphi(N, v)$ for all permutations $\pi$ on $N$ where $\pi v(\pi S)=v(S)$.

Positivity: for all monotonic game $v, \varphi_{i}(N, v) \geq 0$ for all $i \in N$.

Dummy: $v(S)-v(S \backslash i)=v(i)$ for all $S \subset N \Rightarrow \varphi_{i}(N, v)=v(i)$.

These four properties define semivalues as weighted average of marginal contributions:

$$
S E_{i}\left(N, v, p_{n}\right)=\sum_{S \in \mathcal{C}_{i}(N)} p_{n}(s)(v(S)-v(S \backslash i)) \quad(i \in N)
$$

The weights only depend on coalition size and are given by:

$$
p_{n}(s)=\int_{0}^{1} t^{s-1}(1-t)^{n-s} d \mu(t) \quad(s=1, \ldots, n)
$$

where $\mu$ is a probability measure on the interval $[0,1]$. The interpretation is the following. Given a player $i$, pick $t$ at random according to the probability distribution defined by the measure $\mu$ and let players independently join player $i$ with probability $t$. Coalition $S$ containing player $i$ then forms with probability $t^{s-1}(1-t)^{n-s}$. Dubey, Neyman and Weber (1981) prove that the weight vector $p_{n}=\left(p_{n}(1), \ldots, p_{n}(n)\right)$ can be any nonnegative vector satisfying the following normalization condition:

$$
\sum_{s=1}^{n} C_{n-1}^{s-1} p_{n}(s)=1
$$

where $C_{n-1}^{s-1}$ is the number of coalition of size $s$ containing a given player. In an additive game, all players are dummies. Hence, for all weight systems $p_{n}$ satisfying (2), $S E_{i}\left(N, v, p_{n}\right)=v(i)$ for all $i$ if $(N, v)$ is additive.

As far as terminology is concerned, for a given player set, weights define a particular semivalue and that semivalue associates payoff vectors to games. There are two well-known semivalues. The Shapley value is the semivalue defined by: ${ }^{3}$

$$
p_{n}(s)=\frac{(s-1) !(n-s) !}{n !} \quad(s=1, \ldots, t)
$$

The Banzhaf value is the semivalue defined by $p_{n}(s)=1 / 2^{n-1}(s=1, \ldots, t)$. We denote these two semivalues by $S V(N, v)$ and $B V(N, v)$ respectively. Semivalues are generally not efficient. Actually, the Shapley value is the only efficient semivalue.

\footnotetext{
${ }^{3}$ The Shapley value is indeed obtained when $\lambda$ is the Lebesgue measure on $[0,1]$.
} 
A semivalue defined on a game $(N, v)$ by weights $p_{n}$ induces semivalues on the $t$-player subgames $\left(T, v_{T}\right)$ obtained by restricting the characteristic function $v$ to subsets $T \subset N$ : $v_{T}(S)=v(S)$ for all $S \subset T$. From now on, we drop the subscript $T:(T, v)$ will denote the subgame on the subset $T$. The corresponding weights are given by the inverse Pascal triangle formula:

$$
p_{t}(s)=p_{t+1}(s)+p_{t+1}(s+1) \quad(s=1, \ldots, t)
$$

If $p_{n}$ satisfies the normalization condition (2), the weights $p_{t}$ derived from (3) automatically satisfy the normalization condition for all $t \in 1, \ldots, n-1$. In the 3 -player case, the normalization condition reads $p_{3}(1)+2 p_{3}(2)+p_{3}(3)=1$ and the weights of the 2-player subgames are given by $p_{2}(1)=p_{3}(1)+p_{3}(2)$ and $p_{2}(2)=p_{3}(2)+p_{3}(3)$, and satisfy the equation $p_{2}(1)+p_{2}(2)=1$. Moreover, $p_{1}(1)=1$.

Binomial semivalues have been introduced by Puente (2000). They form the subfamily of semivalues defined by $p_{n}(s)=p^{s-1}(1-p)^{n-s}$ for some $p \in[0,1]$. The binomial semivalue of a game $(N, v)$ associated to $p$ is therefore given by:

$$
B E_{i}(N, v, p)=\sum_{S \in \mathcal{C}_{i}(N)} p^{s-1}(1-p)^{n-s}(v(S)-v(S \backslash i)) \quad(i=1, \ldots, n)
$$

For $p=0$ or 1 , we have respectively $B E_{i}(N, v, 0)=v(i)$ and $B E_{i}(N, v, 1)=v(N)-v(N \backslash i) .{ }^{4}$ The Banzhaf value corresponds to the case where $p=1 / 2$. The Shapley value instead is not a binomial semivalue. Binomial semivalues are semivalues where the ratios $p_{n}(s+1) / p_{n}(s)$ are constant, the constant being given by $\lambda=p /(1-p)$. Let's consider the case where these ratios are allowed to vary:

or

$$
p_{n}(1)=\lambda_{1}, \frac{p_{n}(2)}{p_{n}(1)}=\lambda_{2}, \ldots, \frac{p_{n}(n)}{p_{n}(n-1)}=\lambda_{n}
$$

$$
p_{n}(1)=\lambda_{1}, p_{n}(2)=\lambda_{1} \lambda_{2}, \ldots, p_{n}(n)=\lambda_{1} \lambda_{2} \ldots \lambda_{n}
$$

In terms of the $\lambda_{s}$ normalization condition then reads:

$$
\sum_{s=1}^{n} C_{n-1}^{s-1} \lambda_{1} \ldots \lambda_{s}=1
$$

This gives rise to an equivalent formulation of semivalues:

$$
S E_{i}(N, v, \lambda)=\sum_{S \in \mathcal{C}_{i}(N)} \lambda_{1} \lambda_{2} \ldots \lambda_{s}(v(S)-v(S \backslash i)) \quad(i=1, \ldots, n)
$$

This equivalent formulation will be especially useful in solving coalitional rationality.

\footnotetext{
${ }^{4}$ Assuming $0^{0}=1$.
} 
For 3-player games, (4) becomes $p_{3}(1)=\lambda_{1}, p_{3}(2)=\lambda_{1} \lambda_{2}, p_{3}(3)=\lambda_{1} \lambda_{2} \lambda_{3}$ and the normalization condition (5) then reads $\lambda_{1}+2 \lambda_{1} \lambda_{2}+\lambda_{1} \lambda_{2} \lambda_{3}=1$. Weights of the 2-player subgames are given by $p_{2}(1)=\lambda_{1}\left(1+\lambda_{2}\right)$ and $p_{2}(2)=\lambda_{1} \lambda_{2}\left(1+\lambda_{3}\right)$. The weights $\lambda=(1 / 3,1 / 2,2)$ and $\lambda=(1 / 4,1,1)$ produce the Shapley value and Banzhaf value, respectively. They are obtained from the original weights $(1 / 3,1 / 6,1 / 3)$ and $(1 / 4,1 / 4,1 / 4)$. In general, we have:

$$
\lambda_{1}=\frac{1}{n} \text { and } \lambda_{t}=\frac{t-1}{n-t+1} \text { for all } t \geq 2
$$

for the Shapley value. For the Banzhaf value, we have:

$$
\lambda_{1}=\frac{1}{2^{n-1}} \text { and } \lambda_{t}=1 \text { for all } t \geq 2
$$

\section{The inverse problem}

Given a player set $N$, the inverse problem relative to the semivalue defined by weights $p_{n}$ can be stated as follows. Given an arbitrary payoff vector $L \in \mathbb{R}^{n}$, characterize the set

$$
G\left(N, L, p_{n}\right)=\left\{v \in G(N) \mid S E\left(N, v, p_{n}\right)=L\right\}
$$

Given $N$ and $p_{n}$, the operator $S E$ is a linear map from $\mathbb{R}^{2^{n}-1}$ to $\mathbb{R}^{n}$ and $G\left(N, L, p_{n}\right)$ is nonempty: It contains the additive game $\left(N, v_{L}\right)$ defined by $v_{L}(i)=L_{i}$. Notice that, at this stage, non-negativity of $L$ is not imposed. Payoffs will be assumed to have nonnegative components when discussing coalitional rationality.

\subsection{The potential basis}

The potential of a game relative to the Shapley value has been introduced by Hart and MasColell $(1988,1989)$. More generally, a potential relative to a value $\varphi$ is a function $P_{\varphi}: G \rightarrow \mathbb{R}$ such that a player's payoff according to $\varphi$ is equal to his marginal contribution to the grand coalition computed in terms of $P_{\varphi}: \varphi_{i}(N, v)=P_{\varphi}(N, v)-P_{\varphi}(N \backslash i, v)$. The potential of a game, relative to a semivalue, has been introduced by Dragan (1996) and by Calvo and Santos (1997) as follows. Consider the game $(N, v)$ and the semivalue associated to the weight vector $p_{n}$. The potential of the subgame $(T, v)$ is then given by:

$$
P\left(T, v, p_{t}\right)=\sum_{S \subset T} p_{t}(s) v(S) \quad \text { for all } T \subset N
$$

where the weights $p_{t}$ of the subgames are derived by the inverse Pascal triangle rule. Indeed, using (1) and (3), we have:

$$
P\left(N, v, p_{n}\right)-P\left(N \backslash i, v, p_{n-1}\right)=S E_{i}\left(N, v, p_{n}\right) \quad(i=1, \ldots, n)
$$

The most popular bases of the vector space of games on a given set of players are the standard basis and the unanimity basis. The latter was used by Shapley to prove the unicity of his value. The concept of potential may be used to define another basis as shown by Dragan (1991). Given a player set $N$, a basis $\left\{w_{T} \in \mathbb{R}^{2^{n}-1} \mid T \subset N, T \neq \varnothing\right\}$ of $G(N)$ is the potential basis 
relative to the semivalue associated to weights $p_{n}$ if

$$
v=\sum_{T \subset N} P\left(T, v, p_{t}\right) w_{T} \quad \text { for all } v \in G(N)
$$

where the weights $p_{t}$ of the subgames are obtained from (3). In words, the coordinate $T$ of $v$ relative to this basis is the potential of the subgame $(T, v)$. Dragan (2005) proves the following important theorem that specifies the potential basis associated to a given weight system.

Theorem 3.1 Given a player set $N$ and weights $p_{n}$, the family of $2^{n}-1$ games defined by:

$$
w_{T}(S)=\sum_{h=0}^{s-t}(-1)^{h} C_{s-t}^{h} \frac{1}{p_{t+h}(t+h)} \text { for all } S \supset T
$$

and $w_{T}(S)=0$ for all other $S$, is the potential basis relative to the semivalue associated to $p_{n}$.

We observe that $\left\{w_{T}\right\}$ defines a diagonal matrix whose diagonal elements are given by $w_{T}(T)=1 / p_{t}(t)$. Hence, it is a regular matrix and consequently $\left(w_{T}\right)$ is a basis of $G(N)$. We denote it by $W\left(p_{n}\right)$. It will be used to solve the inverse problem. Notice that the weights that appear in the formulation of the basis are all of the form $1 / p_{t}(t)$. The potential basis for the 3player case is given by Table 1. The following theorem specifies the semivalues of the games that are part of the potential basis.

Theorem 3.2 Given weights $p_{n}$ satisfying the normalization condition (2), the semivalues of the potential basis $W\left(p_{n}\right)$ are given by:

$$
\begin{array}{ll}
S E_{i}\left(N, w_{N}, p_{n}\right)=1 & \text { for all } i=1, \ldots, n \\
S E_{i}\left(N, w_{N \backslash i}, p_{n}\right)=-1 & \text { for all } i=1, \ldots, n \\
S E_{i}\left(N, w_{T}, p_{n}\right)=0 & \text { for all other } T \subset N
\end{array}
$$

Proof For a given player $i$ in $S$ and coalition $T$ in $N$, we apply (9) to get:

$$
S E_{i}\left(N, v, w_{T}\right)=P\left(N, w_{T}, p_{n}\right)-P\left(N \backslash i, w_{T}, p_{n-1}\right)
$$

where $P$ is the potential defined by (8). If $T=N$, the first term is equal to 1 while the second is equal to 0 :

$$
\begin{aligned}
& P\left(N, w_{N}, p_{n}\right)=p_{n}(n) \frac{1}{p_{n}(n)}=1 \\
& P\left(N \backslash i, w_{N}, p_{n}\right)=\sum_{S \subset N \backslash i} p_{n-1}(s) w_{N}(S)=0
\end{aligned}
$$


The reverse applies if $T=N \backslash i$ :

$$
\begin{gathered}
P\left(N, w_{N \backslash i}, p_{n}\right)=p_{n}(n-1) \frac{1}{p_{n-1}(n-1)}+p_{n}(n)\left(\frac{1}{p_{n-1}(n-1)}-\frac{1}{p_{n}(n)}\right) \\
=\frac{p_{n}(n-1)+p_{n}(n)}{p_{n-1}(n-1)}-1=0 \\
P\left(N \backslash i, w_{N \backslash i}, p_{n}\right)=p_{n-1}(n-1) \frac{1}{p_{n-1}(n-1)}=1
\end{gathered}
$$

Finally, if $T \neq N$ or $N \backslash i$, both terms are equal to 0 .

\begin{tabular}{|c|c|c|c|c|c|c|c|}
\hline & $\{1\}$ & $\{2\}$ & $\{3\}$ & $\{1,2\}$ & $\{1,3\}$ & $\{2,3\}$ & $\{1,2,3\}$ \\
\hline$w_{1}$ & 1 & 0 & 0 & $1-\frac{1}{p_{2}(2)}$ & $1-\frac{1}{p_{2}(2)}$ & 0 & $1-\frac{2}{p_{2}(2)}+\frac{1}{p_{3}(3)}$ \\
\hline$w_{2}$ & 0 & 1 & 0 & $1-\frac{1}{p_{2}(2)}$ & 0 & $1-\frac{1}{p_{2}(2)}$ & $1-\frac{2}{p_{2}(2)}+\frac{1}{p_{3}(3)}$ \\
\hline$w_{3}$ & 0 & 0 & 1 & 0 & $1-\frac{1}{p_{2}(2)}$ & $1-\frac{1}{p_{2}(2)}$ & $1-\frac{2}{p_{2}(2)}+\frac{1}{p_{3}(3)}$ \\
\hline$w_{12}$ & 0 & 0 & 0 & $\frac{1}{p_{2}(2)}$ & 0 & 0 & $\frac{1}{p_{2}(2)}-\frac{1}{p_{3}(3)}$ \\
\hline$w_{13}$ & 0 & 0 & 0 & 0 & $\frac{1}{p_{2}(2)}$ & 0 & $\frac{1}{p_{2}(2)}-\frac{1}{p_{3}(3)}$ \\
\hline$w_{23}$ & 0 & 0 & 0 & 0 & 0 & $\frac{1}{p_{2}(2)}$ & $\frac{1}{p_{2}(2)}-\frac{1}{p_{3}(3)}$ \\
\hline$w_{123}$ & 0 & 0 & 0 & 0 & 0 & 0 & $\frac{1}{p_{3}(3)}$ \\
\hline
\end{tabular}

Table 3.1: The 3-player potential basis $W\left(p_{3}\right)$

\subsection{Solution to the inverse problem}

The following theorem gives a solution to the inverse problem. It characterizes the set $G\left(N, L, p_{n}\right)$ defined by (7).

Theorem 3.3 The games in $G\left(N, L, p_{n}\right)$ are of the following form:

$$
v=\sum_{T: t \leq n-2} \alpha_{T} w_{T}+\alpha_{N}\left(w_{N}+\sum_{j \in N} w_{N \backslash j}\right)-\sum_{j \in N} L_{j} w_{N \backslash j}
$$

where $\alpha_{N}$ and the $\alpha_{T}$ are $2^{n}-n-1$ arbitrary constants.

Proof Assume that (11) holds. By linearity of the operator $S E$ and Theorem 3.2, we have:

$$
S E_{i}\left(N, v, p_{n}\right)=\alpha_{N}\left(S E_{i}\left(N, w_{N}, p_{n}\right)+S E_{i}\left(N, w_{N \backslash i}, p_{n}\right)\right)-L_{i} S E_{i}\left(N, w_{N \backslash i}, p_{n}\right)=L_{i}
$$


Conversely, by Theorem 3.1 and (10), we know that any game $(N, v)$ can be written in the basis $W\left(p_{n}\right)$ as $\sum \alpha_{T} w_{T}$ where $\alpha_{T}$ is the potential of the restriction of $v$ to the subset $T$. Therefore, following (9), $S E\left(N, v, p_{n}\right)=L$ implies $L_{i}=P\left(N, v, p_{n}\right)-P\left(N \backslash i, v, p_{n}\right)=\alpha_{N}-\alpha_{N \backslash i}$ for all $i$. Hence $\alpha_{N \backslash i}=\alpha_{N}-L_{i}$.

As a consequence of Theorem 3.3, the rank of the linear operator $S E$ is equal to $n$ and the null space $G\left(N, 0, p_{n}\right)$ is a subspace of dimension $2^{n}-n-1$, the nullity of $S E$. The basis of $G\left(N, 0, p_{n}\right)$ is indeed formed by the $2^{n}-n-2$ vectors $\left(w_{T}\right)$ for all $T$ such that $t \leq n-2$, together with the vector $w_{N}+\sum w_{N \backslash i}$. We observe that the games defined by (11) are such that $v(i)=\alpha_{i}$ for all $i$. In the 3 -player case where $N=\{1,2,3\},(11)$ reads:

$$
v(S)=\sum_{j=1}^{3} \alpha_{j} w_{j}(S)+\alpha_{123}\left(w_{123}(S)+\sum_{j=1}^{3} w_{N \backslash j}(S)\right)-\sum_{j=1}^{3} L_{j} w_{N \backslash j}(S) \quad(S \subset N)
$$

Referring to Table 3.1, we have:

$$
\begin{aligned}
& v(i)=\alpha_{i} \quad(i=1,2,3) \\
& v(1,2)=\left(\alpha_{1}+\alpha_{2}\right)\left(1-\frac{1}{p_{2}(2)}\right)+\left(\alpha_{123}-L_{3}\right) \frac{1}{p_{2}(2)} \\
& v(1,3)=\left(\alpha_{1}+\alpha_{3}\right)\left(1-\frac{1}{p_{2}(2)}\right)+\left(\alpha_{123}-L_{2}\right) \frac{1}{p_{2}(2)} \\
& v(2,3)=\left(\alpha_{2}+\alpha_{3}\right)\left(1-\frac{1}{p_{2}(2)}\right)+\left(\alpha_{123}-L_{1}\right) \frac{1}{p_{2}(2)} \\
& v(1,2,3)=\left(\alpha_{1}+\alpha_{2}+\alpha_{3}\right)\left(1-\frac{2}{p_{2}(2)}+\frac{1}{p_{3}(3)}\right) \\
& \quad+\alpha_{123}\left(\frac{3}{p_{2}(2)}-\frac{2}{p_{3}(3)}\right)+\left(\frac{1}{p_{3}(3)}-\frac{1}{p_{2}(2)}\right) \sum L_{i}
\end{aligned}
$$

For the Shapley weights $p_{3}=(1 / 3,1 / 6,1 / 3)$ and $p_{2}=(1 / 2,1 / 2)$, the game defined in $(12)$ becomes:

$$
\begin{aligned}
& v(i)=\alpha_{i} \quad(i=1,2,3) \\
& v(1,2)=-\left(\alpha_{1}+\alpha_{2}\right)+2\left(\alpha_{123}-L_{3}\right) \\
& v(1,3)=-\left(\alpha_{1}+\alpha_{3}\right)+2\left(\alpha_{123}-L_{2}\right) \\
& v(2,3)=-\left(\alpha_{2}+\alpha_{3}\right)+2\left(\alpha_{123}-L_{1}\right) \\
& v(1,2,3)=L_{1}+L_{2}+L_{3}
\end{aligned}
$$


As expected, efficiency applies for Shapley. For the Banzhaf weights $p_{3}=(1 / 4,1 / 4,1 / 4)$ and $p_{2}=(1 / 2,1 / 2)$, it turns out that, with three players, only the worth of the grand coalition changes:

$$
v(1,2,3)=\alpha_{1}+\alpha_{2}+\alpha_{3}-2 \alpha_{123}+2\left(L_{1}+L_{2}+L_{3}\right)
$$

\section{Coalitional rationality}

The payoffs defined by a semivalue may fail to satisfy some or all of the following properties, efficiency, individual rationality and coalitional rationality. Given a player set $N$ and weights $p_{n}$, consider an arbitrary payoff vector L. Question: Can we identify games in $G\left(N, L, p_{n}\right)$ such that $L$ satisfies some or all of the above properties relative to these games? There is a trivial answer: The additive game $\left(N, v_{L}\right)$ satisfies all three properties, independently of the weights. However, we are interested in non-additive games. Individual rationality is no problem. It suffices to impose $\alpha_{i} \leq L_{i}$ for all $i$ in (11). Coalitional rationality instead is a problem. When efficiency holds, the question of coalitional rationality can be raised directly, while in the absence of efficiency, we need an appropriate redefinition of coalitional rationality.

\subsection{The family of almost null games}

From now on, we assume that $L \in \mathbb{R}_{+}^{n}$. To solve the question of coalitional rationality, consider the subsets of games in $G\left(N, L, p_{n}\right)$ defined by $\alpha_{T}=0$ for all coalition $T$ of size $t \leq n-2$ in (11). They are of the following form:

$$
v_{\alpha}=\alpha\left(w_{N}+\sum_{j \in N} w_{N \backslash j}\right)-\sum_{j \in N} L_{j} w_{N \backslash j}
$$

This set of games is called the family of almost null games. It depends on a single parameter, namely $\alpha=\alpha_{N}$. By construction, $\operatorname{SE}\left(N, v_{\alpha}, p_{n}\right)=L$. Referring to Theorem 1 and expressing the components $w_{N}$ and $w_{N \backslash i}$ of the potential basis $W\left(p_{n}\right)$ in terms of the parameters $\lambda_{s}$ we have successively:

$$
\begin{aligned}
& w_{N}(N)=\frac{1}{p_{n}(n)}=\frac{1}{\lambda_{1} \lambda_{2} \ldots \lambda_{n}} \\
& w_{N \backslash i}(N)=\frac{1}{p_{n-1}(n-1)}-\frac{1}{p_{n}(n)}=\frac{-1}{\lambda_{1} \lambda_{2} \ldots \lambda_{n}\left(1+\lambda_{n}\right)} \\
& w_{N \backslash i}(N \backslash i)=\frac{1}{p_{n-1}(n-1)}=\frac{1}{\lambda_{1} \lambda_{2} \ldots \lambda_{n-1}\left(1+\lambda_{n}\right)}
\end{aligned}
$$

and $w_{N}(S)=w_{N \backslash i}(S)=0$ otherwise. The games $\left(N, v_{\alpha}\right)$ are therefore given by:

$$
\begin{aligned}
& v_{\alpha}(N \backslash i)=\frac{\alpha-L_{i}}{\lambda_{1} \lambda_{2} \ldots \lambda_{n-1}\left(1+\lambda_{n}\right)} \\
& v_{\alpha}(N)=\frac{\left(1+\lambda_{n}-n\right) \alpha+\sum L_{j}}{\lambda_{1} \lambda_{2} \ldots \lambda_{n}\left(1+\lambda_{n}\right)}
\end{aligned}
$$


and $v_{\alpha}(S)=0$ for all other $S$.

We have to consider two situations: either efficiency applies, $\sum L_{j}=v_{\alpha}(N)$, or it does not. Efficiency holds for all values of $\alpha$ if and only if the following conditions are satisfied:

$$
\begin{aligned}
& \lambda_{n}=n-1 \\
& n(n-1) \lambda_{1} \lambda_{2} \ldots \lambda_{n-1}=1
\end{aligned} \Leftrightarrow \begin{aligned}
& p_{n}(n)=\frac{1}{n} \\
& p_{n}(n-1)=\frac{1}{n(n-1)}
\end{aligned}
$$

They are verified in the case of the Shapley value. We will see later that, in the 3-player case, they define uniquely the Shapley value, together with the normalization condition (5). From (15), we obtain the following game:

$$
\begin{aligned}
& u_{\alpha}(N \backslash i)=(n-1)\left(\alpha-L_{i}\right) \\
& u_{\alpha}(N)=\sum L_{j}
\end{aligned}
$$

Because only the marginal contributions to the coalitions $N$ and $N \backslash i$ are concerned, its Shapley value coincides with $L: S E\left(N, u_{\alpha}\right)=L$ for all $\alpha$. We now look for the values of the parameter $\alpha$ such that:

$$
u_{\alpha}(N \backslash i) \leq \sum_{j \neq i} L_{j} \text { for all } i \in N
$$

Because $L \geq 0$, the other core conditions are automatically satisfied. Hence, coalitional rationality applies under efficiency if and only if:

$$
\alpha \leq L_{i}+\frac{1}{n-1} \sum_{j \neq i} L_{j} \text { for all } i \in N
$$

If instead efficiency does not apply, coalitional rationality is evaluated with respect to the power game associated to a semivalue. The power game associated to a game $(N, v)$ and weights $p_{n}$ is the game $(N, \pi)$ defined by: ${ }^{5}$

$$
\pi(T)=\sum_{i \in T} S E_{i}\left(T, v, p_{t}\right)=\sum_{i \in T} \sum_{S \in \mathcal{C}_{i}(T)} p_{t}(s)(v(S)-v(S \backslash i)) \quad T \subset N
$$

where the intermediary weights $p_{t}$ are derived from (3) and $\pi(N)=\sum S E_{i}\left(N, v, p_{n}\right)$. Coalitional rationality then holds if $L$ belongs to the core of the power game, called the power core. Following Dragan and Martinez-Legaz (2001), the power game associated to a game $(N, v)$ and weights $p_{n}$ can be conveniently written as:

$$
\pi(T)=\sum_{S \subset T}\left(s p_{t}(s)-(t-s) p_{t}(s+1)\right) v(S)
$$

\footnotetext{
${ }^{5}$ Notice that we have left implicit the dependence of $\pi$ on the game and the weights.
} 
Provided that $p_{n}(n)>0, \pi$ is a bijection from $G(N)$ onto itself. If $S E\left(N, v, p_{n}\right)$ defines an efficient semivalue, it is the Shapley value and we have:

$$
\begin{aligned}
& p_{t}(t)=1 \text { for all } t \\
& s p_{t}(s)-(t-s) p_{t}(s+1)=0 \text { for all } s=1, \ldots, t-1
\end{aligned}
$$

The power game is then the game itself, so that it is a proper extension. Furthermore, the Shapley value of the power game associated to a semivalue is the semivalue itself. In order to define the power game $\left(N, \pi_{\alpha}\right)$ associated to the game $\left(N, v_{\alpha}\right)$ defined by $(15)$, we only need to specify $\pi_{\alpha}(N \backslash i)$ for all $i$. Indeed, $\pi_{\alpha}(N)=\sum L_{j}$ and $\pi_{\alpha}(S)=0$ for all $S \neq N \backslash i$ or $N$. It turns out that the power game coincides with the game $\left(N, u_{\alpha}\right)$ defined by (17) under efficiency, independently of the weights. Indeed, we have:

$$
\begin{aligned}
\pi_{\alpha}(N \backslash i) & =(n-1) p_{n-1}(n-1) v_{\alpha}(N \backslash i)=(n-1)\left(p_{n}(n-1)+p_{n}(n)\right) v_{\alpha}(N \backslash i) \\
& =(n-1) \lambda_{1} \lambda_{2} \ldots \lambda_{n-1}\left(1+\lambda_{n}\right) v_{\alpha}(N \backslash i)=(n-1)\left(\alpha-L_{i}\right)
\end{aligned}
$$

Therefore, the same inequality, namely (18), guarantees coalitional rationality in the absence of efficiency. The following theorem summarizes our findings, knowing that the definition of coalitional rationality depends on efficiency.

Theorem 4.1 Given a player set $N$, a payoff vector $L \in \mathbb{R}_{+}^{n}$ and weights $p_{n}$, the games $\left(N, u_{\alpha}\right)$ defined by (17) are such that $S V\left(N, u_{\alpha}\right)=L$. Furthermore, for $\alpha$ satisfying (18), $L$ belongs to the core of the game $\left(N, u_{\alpha}\right)$ and thereby $L$ satisfies coalitional rationality.

Example 4.1 Consider the 4-player case, with the payoff vector $L=(3,5,9,13)$ and the weights $p_{n}=(1 / 10,2 / 15,1 / 12,1 / 4)$. The corresponding $\lambda_{s}$ are given by $\lambda=(1 / 10,4 / 3,5 / 8,3)$. The weights satisfy (16) and therefore efficiency holds and the game $\left(N, u_{\alpha}\right)$ defined in (17) is then given by:

$$
\begin{aligned}
& u_{\alpha}(1,2,3)=3(\alpha-13) \\
& u_{\alpha}(1,2,4)=3(\alpha-9) \\
& u_{\alpha}(1,3,4)=3(\alpha-5) \\
& u_{\alpha}(2,3,4)=3(\alpha-3) \\
& u_{\alpha}(1,2,3,4)=30
\end{aligned}
$$

Its Shapley value is equal to $L$ for all values of $\alpha$ and belongs to its core for all $\alpha \leq 12$. Now consider a different set of weights, for instance the Banzhaf weights $p_{n}=(1 / 8,1 / 8,1 / 8,1 / 8)$ or $\lambda=(1 / 8,1,1,1)$. 
The game $\left(N, v_{\alpha}\right)$ defined in (15) is then given by:

$$
\begin{aligned}
& v_{\alpha}(1,2,3)=4(\alpha-13) \\
& v_{\alpha}(1,2,4)=4(\alpha-9) \\
& v_{\alpha}(1,3,4)=4(\alpha-5) \\
& v_{\alpha}(2,3,4)=4(\alpha-3) \\
& v_{\alpha}(1,2,3,4)=4(30-2 \alpha)
\end{aligned}
$$

Its Banzhaf value is equal to $L$ and its power game is given by (20). Coalitional rationality therefore follows for all $\alpha \leq 12$.

\subsection{The 3-player case}

The case where $n=3$ is of a particular interest and deserves to be treated apart. Efficiency holds if and only if the semivalue is the Shapley value. Indeed, $\lambda=(1 / 3,1 / 2,2)$ is the unique solution of (16) together with the normalization condition (5). The game $\left(N, v_{\alpha}\right)$ defined in (15) is given by:

$$
\begin{aligned}
& v_{\alpha}(N \backslash i)=2\left(\alpha-L_{i}\right) \text { for } i=1,2,3 \\
& v_{\alpha}(N)=L_{1}+L_{2}+L_{3}
\end{aligned}
$$

It corresponds to the game defined in (13) for $\alpha_{1}=\alpha_{2}=\alpha_{3}=0$. Coalitional rationality then holds for $\alpha$ satisfying (18):

$$
\alpha \leq \frac{1}{2} \operatorname{Min}\left(2 L_{1}+L_{2}+L_{3}, L_{1}+2 L_{2}+L_{3}, L_{1}+L_{2}+2 L_{3}\right)
$$

Now consider the case where $\lambda=(1 / 4,1,1)$ which defines the Banzhaf value. Only the worth of the grand coalition changes:

$$
v_{\alpha}(N)=2\left(L_{1}+L_{2}+L_{3}-\alpha\right)
$$

It corresponds to the game defined in (14) for $\alpha_{1}=\alpha_{2}=\alpha_{3}=0$. Looking at $v_{\alpha}(N)$, we observe that it is possible to obtain both efficiency and coalitional rationality for the Banzhaf value by setting $\alpha=(1 / 2) \sum L_{i}$. The resulting game $(N, u)$ is given by:

$$
\begin{aligned}
& u(1,2)=L_{1}+L_{2}-L_{3} \\
& u(1,3)=L_{1}+L_{3}-L_{2} \\
& u(2,3)=L_{2}+L_{3}-L_{1} \\
& u(1,2,3)=L_{1}+L_{2}+L_{3}
\end{aligned}
$$


Computation confirms that the Shapley value and the Banzhaf value both coincides with $L$. Furthermore, because $\alpha$ satisfies (18), $L$ belongs to the core of the game $(N, u)$. This is a particularity of 3-player games that does not carry over to a larger $n$. In the 4-player case, with Shapley weights $\lambda=(1 / 4,1 / 3,1,3), L$ satisfy coalitional rationality if:

$$
\alpha \leq \frac{1}{4} \operatorname{Min}\left(4 L_{1}+L_{2}+L_{3}+L_{4}, L_{1}+4 L_{2}+L_{3}+L_{4}, L_{1}+L_{2}+4 L_{3}+L_{4}, L_{1}+L_{2}+L_{3}+4 L_{4}\right)
$$

With Banzhaf weights $\lambda=(1 / 8,1,1,1)$, we get:

$$
\begin{aligned}
& v_{\alpha}(N \backslash i)=4\left(\alpha-L_{i}\right) \\
& v_{\alpha}(N)=4\left(\sum L_{j}-2 \alpha\right)
\end{aligned}
$$

Setting $\alpha=(3 / 8) \sum L_{j}$ the game becomes:

$$
\begin{aligned}
& u(N \backslash i)=\frac{3}{2} \sum L_{j}-4 L_{i} \\
& u(N)=\sum L_{j}
\end{aligned}
$$

By construction, $L$ equals the Banzhaf value. However, coalitional rationality cannot be obtained in general as the following example shows.

Example 4.2 Let $L=(3,5,9,13)$. In the case of Shapley, $L$ satisfies coalitional rationality if $\alpha \leq 9.75$. For instance, setting $\alpha=9$, we obtain the following game:

$$
\begin{aligned}
& u(123)=-12 \\
& u(124)=0 \\
& u(134)=12 \\
& u(234)=18 \\
& u(1234)=30
\end{aligned}
$$

In the case of Banzhaf, setting $\alpha=45 / 4$, we obtain the following game:

$$
\begin{aligned}
& u(1,2,3)=-7 \\
& u(1,2,4)=9 \\
& u(1,3,4)=25 \\
& u(2,3,4)=33 \\
& u(1,2,3,4)=30
\end{aligned}
$$

By construction its Banzhaf index equals $L$. It fails coalitional rationality: the coalitions $\{2,3,4\}$ and $\{1,3,4\}$ do not get their worth. The core is actually empty. 


\section{Concluding remarks}

The quest for games that reproduce any given nonnegative payoff vector that belongs to their cores can be used to answer the following question: How to modify a game with an empty core in such a way that its Shapley value satisfies coalitional rationality. This is illustrated by the following example.

Example 5.1 Consider the 3-player simple majority game defined by

$$
\begin{aligned}
& v(1)=v(2)=v(3)=0 \\
& v(1,2)=v(1,3)=v(2,3)=1 \\
& v(1,2,3)=1
\end{aligned}
$$

This game has an empty core. It is a symmetric game and therefore the Shapley value is the egalitarian payoff that allocates $1 / 3$ to each player. With Shapley weights, the games in the family of almost null games are given by (13):

$$
\begin{aligned}
& u_{\alpha}(1)=u_{\alpha}(2)=u_{\alpha}(3)=0 \\
& u_{\alpha}(1,2)=u_{\alpha}(1,3)=u_{\alpha}(2,3)=2\left(\alpha-\frac{1}{3}\right) \\
& u_{\alpha}(1,2,3)=1
\end{aligned}
$$

Setting $\alpha=2 / 3$ to satisfy (18), we obtain the following game:

$$
\begin{aligned}
& u(1)=u(2)=u(3)=0 \\
& u(1,2)=u(1,3)=u(2,3)=\frac{2}{3} \\
& u(1,2,3)=1
\end{aligned}
$$

Its core is nonempty and reduces to the singleton $(1 / 3,1 / 3,1 / 3)$.

Semivalues are symmetric in the sense that players contributing identically to coalitions obtain the same amount. The inverse problem for efficient and asymmetric values has been addressed by Dragan (2012). Asymmetric values include the weighted Shapley values, random order values and values derived from arbitrary distributions of Harsanyi dividends. ${ }^{6}$ The related question of coalitional rationality will be the object of further research.

\footnotetext{
${ }^{6}$ See Dehez (2017) for a synthesis.
} 


\section{References}

Banzhaf, J.F. (1965), Weighted voting doesn't work. A mathematical analysis, Rutgers Law Review 19, 317-343.

Calvo E. and Santos, J.C. (1997), Potentials in cooperative TU games, Mathematical Social Sciences 34, 173-190.

Dehez, P. (2017), On Harsanyi dividends and asymmetric values, International Game Theory Review 19.

Dragan, I. (1991), The potential basis and the weighted Shapley value, Libertas Mathematica $11,139-150$.

Dragan, I. (1996), New mathematical properties of the Banzhaf value, European Journal of Operational Research 95, 451-463.

Dragan, I. (2005), On the inverse problem for semivalues of cooperative TU-games, International Journal of Pure and Applied Mathematics 22, 539-555.

Dragan, I. (2012), On the inverse problem for multiweigthed Shapley values of cooperative TU-games, International Journal of Pure and Applied Mathematics 75, 279-287.

Dragan, I. (2014), Coalitional rationality and the inverse problem for binomial semivalues, in Contributions to Game Theory and Management (L. Petrosjan, N. Zenkevich, eds.). St. Petersburg University, Russia, vol.VII, 24-33.

Dragan, I. and Martinez-Legas, J.E. (2001), On the Semivalues and the power core of cooperative TU-games, American Journal of Operations Research 3, 127-139.

Dubey, P., Neyman A. and Weber R.J. (1981), Value theory without efficiency, Mathematics of Operations Research 6, 122-128.

Gillies, D.B. (1953), Some theorems on $n$-person games, Ph.D. Thesis, Princeton University.

Hart, S. and Mas-Colell, A. (1988), The potential of the Shapley value, in The Shapley Value (A.E. Roth, ed.), Cambridge University Press, 127-138.

Hart, S. and Mas-Colell, A. (1989), Potential, value and consistency, Econometrica 57, 589614.

Izawa, Y. and Takahashi, W. (1998), The coalitional rationality of the Shapley value, Journal of Mathematical Analysis and Applications 220, 597-602.

Kleinberg, N. and Weiss, J. (1985), Equivalent $n$-person games and the null space of the Shapley value, Mathematics of Operations Research 10, 233-243.

Maschler, M., Solan, E. and Zamir, S. (2013), Game theory, Cambridge University Press, Cambridge.

Owen, G. (1995), Game Theory ( $3^{\text {rd }}$ ed.), Academic Press, San Diego.

Puente, A.M. (2000), Contributions to the representability of simple games and to the calculus of solutions for this class of games, Ph.D. Thesis, Technical University of Catalonia, Spain.

Shapley, L.S. (1951), Notes on the $n$-person game II: The value of an $n$-person game, RAND Research Memorandum 670.

Shapley, L.S. (1953), A value for n-person games, Annals of Mathematical Studies 28, Princeton University Press, 307-317. 